\title{
Fourier-Mukai partners of elliptic ruled surfaces
}

\author{
Hokuto Uehara
}

\begin{abstract}
We study Fourier-Mukai partners of elliptic ruled surfaces. We also describe the autoequivalence group of the derived categories of ruled surfaces with an elliptic fibration, by using [Ue15].
\end{abstract}

\section{Introduction}

\subsection{Motivations and results}

Let $X$ be a smooth projective variety over $\mathbb{C}$ and $D(X)$ the bounded derived category of coherent sheaves on $X$. If $X$ and $Y$ are smooth projective varieties with equivalent derived categories, then we call $X$ and $Y$ FourierMukai partners. We denote by $\operatorname{FM}(S)$ the set of isomorphism classes of Fourier-Mukai partner of $X$ :

$$
\operatorname{FM}(X):=\{Y \text { smooth projective varieties } \mid D(X) \cong D(Y)\} / \cong .
$$

It is an interesting problem to determine the set $\operatorname{FM}(X)$ for a given $X$. There are several known results in this direction. For example, Bondal and Orlov show that if $K_{X}$ or $-K_{X}$ is ample, then $X$ can be entirely reconstructed from $D(X)$, namely $\operatorname{FM}(X)=\{X\}$ ([BO95]). To the contrary, there are examples of non-isomorphic varieties $X$ and $Y$ having equivalent derived categories. For example, in dimension 2, if $\operatorname{FM}(X) \neq\{X\}$, then $X$ is a K3 surface, an abelian surface or a relatively minimal elliptic surface with non-zero Kodaira dimension ( $\mathrm{BM} 01$, , Ka02]).

By the classification of surfaces, relatively minimal elliptic surfaces with negative Kodaira dimension are either rational elliptic surfaces or elliptic ruled surfaces. In [Ue04, Ue11, the author studies the set $\operatorname{FM}(S)$ of rational elliptic surfaces $S$. In this paper, we describe the set $\operatorname{FM}(S)$ of elliptic ruled surfaces $S$ :

Theorem 1.1. Let $f: S=\mathbb{P}(\mathcal{E}) \rightarrow E$ be a $\mathbb{P}^{1}$-bundle over an elliptic curve $E$, and $\mathcal{E}$ be a normalized locally free sheaf of rank 2. If $|\operatorname{FM}(S)| \neq 1$, there is a degree 0 line bundle $\mathcal{L} \in \hat{E}:=\operatorname{Pic}^{0} E$ of order $m>4$ such that $\mathcal{E}=\mathcal{O}_{E} \oplus \mathcal{L}$. Furthermore in this case, we have

$$
\operatorname{FM}(S)=\left\{\mathbb{P}\left(\mathcal{O}_{E} \oplus \mathcal{L}^{i}\right) \mid i \in(\mathbb{Z} / m \mathbb{Z})^{*}\right\} / \cong .
$$


This set consists of $\varphi(m) /\left|H_{\hat{\hat{E}}}^{\mathcal{L}}\right|$ elements. Here, $\varphi$ is the Euler function, and $H_{\hat{E}}^{\mathcal{L}}$ is a group defined in 9 2.2 with $\left|H_{\hat{E}}^{\mathcal{L}}\right|=2,4$ or 6 , depending on the choice of $E$ and $\mathcal{L}$.

As an application, in 44 , we describe the autoequivalence group of the derived categories of certain elliptic ruled surfaces by using the result in Ue15.

\subsection{Notation and conventions}

All varieties will be defined over $\mathbb{C}$, unless stated otherwise. A point on a variety will always mean a closed point. By an elliptic surface, we will always mean a smooth projective surface $S$ together with a smooth projective curve $C$ and a relatively minimal morphism $\pi: S \rightarrow C$ whose general fiber is an elliptic curve. Here a relatively minimal morphism means a morphism whose fibers contains no $(-1)$-curves. Such a morphism $\pi$ is called an elliptic fibration.

For an elliptic curve $E$ and some positive integer $m$, we denote the set of points of order $m$ by ${ }_{m} E$. Furthermore, we denote the dual elliptic curve, namely the group scheme $\operatorname{Pic}^{0} E$ of line bundles on $E$ of degree 0 , by $\hat{E}$, and the group of automorphisms of $E$ fixing the origin by $\mathrm{Aut}_{0} E$.

$D(X)$ denotes the bounded derived category of coherent sheaves on an algebraic variety $X$, and Auteq $D(X)$ denotes the group of isomorphism classes of $\mathbb{C}$-linear exact autoequivalences of a $\mathbb{C}$-linear triangulated category $D(X)$.

Let $X$ and $Y$ be smooth projective varieties. For an object $\mathcal{P} \in D(X \times$ $Y$ ), we define an exact functor $\Phi^{\mathcal{P}}$, called an integral functor, to be

$$
\Phi^{\mathcal{P}}:=\mathbb{R} p_{Y *}\left(\mathcal{P} \stackrel{\mathbb{L}}{\otimes} p_{X}^{*}(-)\right): D(X) \rightarrow D(Y),
$$

where we denote the projections by $p_{X}: X \times Y \rightarrow X$ and $p_{Y}: X \times Y \rightarrow Y$. By the result of Orlov ([0r97]), for a fully faithful functor $\Phi: D(X) \rightarrow D(Y)$, there is an object $\mathcal{P} \in D(X \times Y)$, unique up to isomorphism, such that $\Phi \cong \Phi^{\mathcal{P}}$. If an integral functor $\Phi^{\mathcal{P}}$ is an equivalence, it is called a FourierMukai transform.

\subsection{Acknowledgments}

The author is supported by the Grants-in-Aid for Scientific Research (No.23340011). Main part of this paper was written during his staying at the Max-Planck Institute for Mathematics, in the period from April to September 2014. He appreciates the hospitality. 


\section{Preliminaries}

\subsection{Fourier-Mukai transforms on elliptic surfaces}

Bridgeland, Maciocia and Kawamata show in BM01, Ka02] that if a smooth projective surface $S$ has a non-trivial Fourier-Mukai partner $T$, that is $|\operatorname{FM}(S)| \neq 1$, then both of $S$ and $T$ are abelian varieties, K3 surfaces or elliptic surfaces with non-zero Kodaira dimension. We consider the last case in more detail. Many results in this subsection are shown in Br98. Readers are recommended to refer to the original paper [Br98].

Let $\pi: S \rightarrow C$ be an elliptic surface. For an object $E$ of $D(S)$, we define the fiber degree of $E$ as

$$
d(E)=c_{1}(E) \cdot F,
$$

where $F$ is a general fiber of $\pi$. Let us denote by $r(E)$ the rank of $E$ and by $\lambda_{S}$ the highest common factor of the fiber degrees of objects of $D(S)$. Equivalently, $\lambda_{S}$ is the smallest number $d$ such that there is a $d$-section of $\pi$. Consider integers $a$ and $b$ with $a>0$ and $b$ coprime to $a \lambda_{S}$. Then, there exists a smooth, 2-dimensional component $J_{S}(a, b)$ of the moduli space of pure dimension one stable sheaves on $S$, the general point of which represents a rank $a$, degree $b$ stable vector bundle supported on a smooth fiber of $\pi$. There is a natural morphism $J_{S}(a, b) \rightarrow C$, taking a point representing a sheaf supported on the fiber $\pi^{-1}(x)$ of $S$ to the point $x$. This morphism is a relatively minimal elliptic fibration. Furthermore, there is a universal sheaf on $\mathcal{U}$ on $J_{S}(a, b) \times S$ such that the integral functor $\Phi^{\mathcal{U}}$ is a Fourier-Mukai transform.

Put $J_{S}(b):=J_{S}(1, b)$. Obviously, we have $J_{S}(1) \cong S$. As is shown in [BM01, Lemma 4.2], there is also an isomorphism

$$
J_{S}(a, b) \cong J_{S}(b) .
$$

Theorem 2.1 (Proposition 4.4 in [BM01]). Let $\pi: S \rightarrow C$ be an elliptic surface and $T$ a smooth projective variety. Assume that the Kodaira dimension $\kappa(S)$ is non-zero. Then the following are equivalent.

(i) $T$ is a Fourier-Mukai partner of $S$.

(ii) $T$ is isomorphic to $J_{S}(b)$ for some integer $b$ with $\left(b, \lambda_{S}\right)=1$.

There are natural isomorphisms

$$
J_{S}(b) \cong J_{S}\left(b+\lambda_{S}\right) \cong J_{S}(-b)
$$

(see [BM01, Remark 4.5]). Therefore, we can define the subset

$$
H_{S}:=\left\{b \in\left(\mathbb{Z} / \lambda_{S} \mathbb{Z}\right)^{*} \mid J_{S}(b) \cong S\right\}
$$

of the multiplicative group $\left(\mathbb{Z} / \lambda_{S} \mathbb{Z}\right)^{*}$. We can see that $H_{S}$ is a subgroup of $\left(\mathbb{Z} / \lambda_{S} \mathbb{Z}\right)^{*}$, and there is a natural one-to-one correspondence between the set $\operatorname{FM}(S)$ and the quotient group $\left(\mathbb{Z} / \lambda_{S} \mathbb{Z}\right)^{*} / H_{S}$ (see [Ue15, $\left.\S 2.6\right]$ ). 
Claim 2.2. When $\lambda_{S} \leq 4$, we have $|\operatorname{FM}(S)|=1$.

Proof. When $\lambda_{S} \leq 2,\left(\mathbb{Z} / \lambda_{S} \mathbb{Z}\right)^{*}$ is trivial and hence, $\operatorname{FM}(S)=\{S\}$. For $\lambda_{S}>2$ and $b \in\left(\mathbb{Z} / \lambda_{S} \mathbb{Z}\right)^{*}$, we have $b \neq \lambda_{S}-b$ in $\left(\mathbb{Z} / \lambda_{S} \mathbb{Z}\right)^{*}$, and hence, the isomorphisms (1) yield

$$
|\operatorname{FM}(S)| \leq \varphi\left(\lambda_{S}\right) / 2
$$

where $\varphi$ is the Euler function. This inequality implies $|\operatorname{FM}(S)|=1$ for $\lambda_{S} \leq 4$.

In general, it is not easy to describe the group $H_{S}$, equivalently to describe the set $\operatorname{FM}(S)$, concretely. However, even if $\lambda_{S} \geq 5$, there are several examples in which we can compute the cardinality of the set $\operatorname{FM}(S)$ (see [Ue11, Example 2.6]).

\subsection{Some technical lemmas on elliptic curves}

Let $F$ be an elliptic curve. For points $x_{1}, x_{2} \in F$, to distinguish the summations as divisors and as elements in the group scheme $F$, we denote by $x_{1} \oplus x_{2}$ the sum of them by the operation of $F$, and

$$
i \cdot x_{1}:=x_{1} \oplus \cdots \oplus x_{1} \quad(i \text { times }) .
$$

We also denote by

$$
i x_{1}:=x_{1}+\cdots+x_{1} \quad(i \text { times })
$$

the divisor on $F$ of degree $i$. As is well-known, there is a group scheme isomorphism

$$
F \rightarrow \hat{F} \quad x \mapsto \mathcal{O}_{F}(x-O),
$$

where $O$ is the origin of $F$. If we identify $\hat{F}$ and $F$ by (2), so called the normalized Poincare bundle $\mathcal{P}_{0}$ on $F \times F$ is defined by

$$
\mathcal{P}_{0}:=\mathcal{O}_{F \times F}\left(\Delta_{F}-F \times O-O \times F\right),
$$

where $\Delta_{F}$ is the diagonal of $F$ in $F \times F$. It satisfies that

$$
\left.\left.\mathcal{P}_{0}\right|_{F \times x} \cong \mathcal{P}_{0}\right|_{x \times F} \cong \mathcal{O}_{F}(x-O)
$$

for a point $x \in F$.

Let us fix an element $a \in{ }_{m} F$ with a positive integer $m$. Let us denote by $E$ the quotient variety $F /\langle a\rangle$, by

$$
q: F \rightarrow E
$$

the quotient morphism, and by

$$
\hat{q}: \hat{E} \rightarrow \hat{F}
$$


the dual isogeny of $q$. Define a subgroup of $(\mathbb{Z} / m \mathbb{Z})^{*}$ as

$$
H_{F}^{a}:=\left\{k \in(\mathbb{Z} / m \mathbb{Z})^{*} \mid \exists \phi \in \operatorname{Aut}_{0} F \text { such that } \phi(a)=k \cdot a\right\} .
$$

Recall that

- $F \cong \mathbb{C} /(\mathbb{Z}+\sqrt{-1} \mathbb{Z}), \operatorname{Aut}_{0} F=\{ \pm 1, \pm \sqrt{-1}\}$ when $j(F)=1728$,

- $F \cong \mathbb{C} /(\mathbb{Z}+\omega \mathbb{Z})$, Aut $_{0} F=\left\{ \pm 1, \pm \omega, \pm \omega^{2}\right\}$ when $j(F)=0$, and

- $\operatorname{Aut}_{0} F=\{ \pm 1\}$ when $j(F) \neq 0,1728$.

Here $j(F)$ is the $j$-invariant of $F$, and we put $\omega=\frac{-1+\sqrt{-3}}{2}$. We use the following technical lemmas in the proof of Theorem 1.1

Lemma 2.3. Suppose that $m>3$. Then exactly one of the following three cases for $F$ and $a \in{ }_{m} F$ occurs.

(i) The equality $H_{F}^{a}=\{ \pm 1\}$ holds.

(ii) We have $j(F)=1728$, and there is an integer $n$ such that $m$ divides $n^{2}+1$. (Note that this condition implies that $\pm n \in(\mathbb{Z} / m \mathbb{Z})^{*}$.) Moreover, the point $a \in F$ is an element in the subgroup

$$
\left\langle\frac{n}{m}+\frac{1}{m} \sqrt{-1}\right\rangle
$$

of $F \cong \mathbb{C} /(\mathbb{Z}+\sqrt{-1} \mathbb{Z})$, and the equality $H_{F}^{a}=\{ \pm 1, \pm n\}$ holds.

(iii) We have $j(F)=0$, and there is an integer $n$ such that $m$ divides $n^{2}+n+1$. (Note that this condition implies that $\pm n \in(\mathbb{Z} / m \mathbb{Z})^{*}$.) Moreover, the point $a \in F$ is an element in the subgroup

$$
\left\langle\frac{n+1}{m}+\frac{1}{m} \omega\right\rangle
$$

of $F \cong \mathbb{C} /(\mathbb{Z}+\omega \mathbb{Z})$, and the equality $H_{F}^{a}=\left\{ \pm 1, \pm n, \pm n^{2}\right\}$ holds.

Proof. When $j(F) \neq 0,1728$, obviously the case (i) occurs.

Next, let us consider the case $j(F)=1728$. Put $a=\frac{x}{m}+\frac{y}{m} \sqrt{-1}$ for some $x, y \in \mathbb{Z}$, and suppose first that an equality

$$
\sqrt{-1} a=n \cdot a
$$

holds for some $n \in \mathbb{Z}$. Then we have

$$
n x \equiv-y, \quad n y \equiv x \quad(\bmod m) .
$$

Hence, we know that $a=\frac{n y}{m}+\frac{y}{m} \sqrt{-1}$, and since the order of $a$ in $F$ is $m, m$ and $y$ are coprime. The coprimality and the equations (4) yield that 
$m$ divides $n^{2}+1$. The coprimality also implies that the subgroups $\langle a\rangle$ and $\left\langle\frac{n}{m}+\frac{1}{m} \sqrt{-1}\right\rangle$ coincide. We know from Aut $_{0} F=\{ \pm 1, \pm \sqrt{-1}\}$ that $H_{F}^{a}=\{ \pm 1, \pm n\}$ holds.

In the case (iii), the proof is similar.

It follows from the conditions on $m$ and $n$ that $\left|H_{F}^{a}\right|=2,4$ and 6 in the case (i), (ii) and (iii) respectively, hence the two cases do not occur at the same time.

Recall that

$$
\begin{aligned}
H_{\hat{E}}^{\mathcal{L}} & :=\left\{k \in(\mathbb{Z} / m \mathbb{Z})^{*} \mid \exists \hat{\psi} \in \operatorname{Aut}_{0} \hat{E} \text { such that } \hat{\psi}(\mathcal{L})=\mathcal{L}^{k}\right\} \\
& =\left\{k \in(\mathbb{Z} / m \mathbb{Z})^{*} \mid \exists \psi \in \text { Aut }_{0} E \text { such that } \psi^{*} \mathcal{L}=\mathcal{L}^{k}\right\}
\end{aligned}
$$

for a line bundle $\mathcal{L} \in{ }_{m} \hat{E}$.

Lemma 2.4. In each case of Lemma 2.3, the equality $H_{F}^{a}=H_{\hat{E}}^{\mathcal{L}}$ holds for any $\mathcal{L} \in{ }_{m} \hat{E}$ with $\operatorname{ker} \hat{q}=\langle\mathcal{L}\rangle$. (In particular, there is an isomorphism $F \cong E$ in the cases (ii) and (iii), since their $j$-invariants coincide.)

Proof. Let us consider the case (ii) first. Let $L$ be the lattice generated by 1 and $\sqrt{-1}$ in $\mathbb{C}$ so that $F$ with $j(F)=1728$ is isomorphic to $\mathbb{C} / L$. Moreover, the elliptic curve $E=F /\langle a\rangle$ is isomorphic to $\mathbb{C} /(L+\langle a\rangle)$. We can see that the lattice $L+\langle a\rangle$ is preserved by the complex multiplication by $\sqrt{-1}$. (Hence, $j(E)=1728$, which implies $F \cong E$.) It turns out that the quotient morphism

$$
q: F \cong \mathbb{C} / L \rightarrow E \cong \mathbb{C} /(L+\langle a\rangle)
$$

induced by the inclusion $L \hookrightarrow L+\langle a\rangle$ is compatible with the complex multiplication by $\sqrt{-1}$.

Take an element $\frac{1}{m} \in \mathbb{C} / L(\cong F)$, and put

$$
a:=\frac{n y}{m}+\frac{y}{m} \sqrt{-1}
$$

for the integer $n$ in (ii) and some $y \in(\mathbb{Z} / m \mathbb{Z})^{*}$. We define $\mathcal{L}^{\prime}$ to be the element in $\hat{E}$ corresponding to $q\left(\frac{1}{m}\right) \in E$ via $E \cong \hat{E}$. Then we have

$$
\sqrt{-1} q\left(\frac{1}{m}\right)=q\left(\frac{1}{m} \sqrt{-1}\right)=q\left(y^{-1} a-\frac{n}{m}\right)=-n q\left(\frac{1}{m}\right),
$$

and this implies the equality $H_{F}^{a}=\{ \pm 1, \pm n\}=H_{\hat{E}}^{\mathcal{L}^{\prime}}$. We can also see that

$$
\left\langle a, \frac{1}{m}\right\rangle=\left\langle\frac{\sqrt{-1}}{m}, \frac{1}{m}\right\rangle=\operatorname{ker}[m],
$$

where $[m]$ is the multiplication map by $m$. Recall that $[m]=\hat{q} \circ q$ and $\operatorname{ker} q=\langle a\rangle$. Consequently, we have $\operatorname{ker} \hat{q}=\left\langle\mathcal{L}^{\prime}\right\rangle$. For any $\mathcal{L} \in{ }_{m} \hat{E}$ with ker $\hat{q}=\langle\mathcal{L}\rangle$, the equality $H_{\hat{E}}^{\mathcal{L}}=H_{\hat{E}}^{\mathcal{L}^{\prime}}$ holds, which gives the assertion. 
The proof of the case (iii) is similar.

Next let us take an element $\mathcal{L} \in \operatorname{ker} \hat{q}$, and suppose that $\left|H_{\hat{E}}^{\mathcal{L}}\right|=4$ or 6 , namely the case (ii) or (iii) occurs for $\hat{E}$ and $\mathcal{L} \in{ }_{m} \hat{E}$. Then we have already shown above that $H_{F}^{a}=H_{\hat{E}}^{\mathcal{L}}$ (just by replacing the roles of $\hat{E}$ and $F)$. Consequently, in the case (i), we again obtain the assertion.

\subsection{Elliptic ruled surfaces over a field of arbitrary character- istic}

In this subsection, we refer a result which is needed in the proof of Theorem 1.1. The results and notation here over a positive characteristic field are not logically needed in this paper, but we leave them to explain a background of Problem 4.1.

Let $k$ be an algebraically closed field of characteristic $p \geq 0$. Suppose that $E$ is an elliptic curve defined over $k, \mathcal{E}$ is a normalized, in the sense of [Ha77, V. $\S 2$ ], locally free sheaf of rank 2 on $E$, and $f: S=\mathbb{P}(\mathcal{E}) \rightarrow E$ is a $\mathbb{P}^{1}$ bundle on $E$. Set $e:=-\operatorname{deg} \mathcal{E}$. Then we can see that $e=0$ or -1 if $-K_{S}$ is nef, and in particular, if $S$ has an elliptic fibration $\pi: S \rightarrow \mathbb{P}^{1}$. Furthermore, when the locally free sheaf $\mathcal{E}$ is decomposable and $e=0$, it turns out that $\mathcal{E}=\mathcal{O}_{E} \oplus \mathcal{L}$ for some $\mathcal{L} \in \hat{E}$. When $e=-1$, it is indecomposable (see Ha77, $\mathrm{V}$. Theorem 2.12]).

We use the following result to describe the set $\operatorname{FM}(S)$ for elliptic ruled surfaces $S$ in Theorem 1.1,

Theorem 2.5 ([To11]). We use the above notation.

(i) For $e=0, S$ has an elliptic fibration in the cases (i-1), (i-2) and (i-5). Moreover, we have the following:

\begin{tabular}{|c|c|c|c|}
\hline & $\mathcal{E}$ & singular fibers & $p$ \\
\hline \hline$(i-1)$ & $\mathcal{O}_{E} \oplus \mathcal{O}_{E}$ & no singular fibers & $p \geq 0$ \\
\hline$(i-2)$ & $\mathcal{O}_{E} \oplus \mathcal{L}$, ord $\mathcal{L}=m>1$ & $2 \times{ }_{m} \mathrm{I}_{0}$ & $p \geq 0$ \\
\hline$(i-3)$ & $\mathcal{O}_{E} \oplus \mathcal{L}$, ord $\mathcal{L}=\infty$ & & $p \geq 0$ \\
\hline$(i-4)$ & indecomposable & & $p=0$ \\
\hline$(i-5)$ & indecomposable & ${ }_{p} \mathrm{I}_{0}$ (a wild fiber $)$ & $p>0$ \\
\hline
\end{tabular}

(ii) Suppose that $e=-1$ and $p \neq 2$. Then, $S$ has an elliptic fibration with 3 singular fibers of type ${ }_{2} \mathrm{I}_{0}$.

Maruyama also considers the condition that elliptic ruled surfaces have an elliptic fibration [Ma71, Theorem 4], in terms of elementary transformations of ruled surfaces.

Remark 2.6. Let $C_{0}$ be a section of $f$ satisfying $\mathcal{O}_{S}\left(C_{0}\right) \cong \mathcal{O}_{\mathbb{P}(\mathcal{E})}(1)$ (see [Ha77, p. 373]), $F$ be a general fiber of $\pi$, and $F_{f}$ a fiber of $f$. Then [Ha77, 
V. Corollary 2.11] tells us that

$$
K_{S} \equiv-2 C_{0}-e F_{f},
$$

and by the canonical bundle formula of elliptic fibrations, we have

$$
K_{S} \equiv-\frac{2}{m} F
$$

in the case (i-2), and

$$
K_{S} \equiv-\frac{1}{2} F
$$

in the case (ii). Then, we can see that $F \cdot F_{f}=m$ (resp. $F \cdot C_{0}=2$ ), and hence, we have $\lambda_{S}=m$ (resp. $\left.\lambda_{S}=2\right)$ in (i-2) (resp. in (ii)).

\section{Proof of Theorem 1.1}

We give the proof of Theorem 1.1 in the last of this section. Before giving the proof, we need several claims.

Let us take a cyclic group $G=\mathbb{Z} / m \mathbb{Z}$ for an integer $m>1$ and a generator $g$ of $G$. For integers $i \in(\mathbb{Z} / m \mathbb{Z})^{*}$, define representations

$$
\rho_{\mathbb{P}^{1}}: G \rightarrow \operatorname{Aut}\left(\mathbb{P}^{1}\right) \quad \text { as } \quad \rho_{\mathbb{P}^{1}}(g)(y)=\zeta y,
$$

and

$$
\rho_{F, i}: G \rightarrow \operatorname{Aut}(F) \quad \text { as } \quad \rho_{F, i}(g)(x)=T_{i \cdot a} x,
$$

where $a$ is an element of ${ }_{m} F, T_{a}$ is the translation by $a$ and $\zeta$ is a primitive $m$-th root of unity in $\mathbb{C}$. Let us consider the diagonal action

$$
\rho_{i}\left(:=\rho_{F, i} \times \rho_{\mathbb{P}^{1}}\right): G \rightarrow \operatorname{Aut}\left(F \times \mathbb{P}^{1}\right)
$$

induced by $\rho_{\mathbb{P}^{1}}$ and $\rho_{F, i}$. Set

$$
S_{i}:=\left(F \times \mathbb{P}^{1}\right) /{ }_{i} G,
$$

the quotient of $F \times \mathbb{P}^{1}$ by the action $\rho_{i}$. Then we have the following commutative diagram:

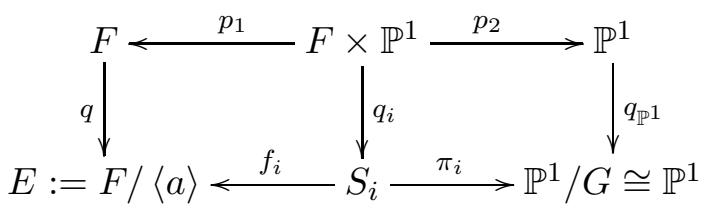

Here, every vertical arrow is the quotient morphism of the action of $G$. We can readily see that $f_{i}$ is a $\mathbb{P}^{1}$-bundle and $\pi_{i}$ is an elliptic fibration. Note that the quotient morphism $q$ does not depend on the choice of $i$, and that 
the left square in (6) is a fiber product. We can also see that $\pi_{i}$ has exactly two multiple fibers of type ${ }_{m} \mathrm{I}_{0}$ over the branch points $q_{\mathbb{P}^{1}}(0), q_{\mathbb{P}^{1}}(\infty)$ of $q_{\mathbb{P}^{1}}$, and it fits into the case (i-2) in Theorem 2.5. Consequently, there is a line bundle $\mathcal{L}_{i} \in{ }_{m} \hat{E}$ such that

$$
S_{i} \cong \mathbb{P}\left(\mathcal{O}_{E} \oplus \mathcal{L}_{i}\right)
$$

for each $i$. Furthermore, because the left square in (6) is a fiber product, we have $q^{*} \mathcal{L}_{i}=\mathcal{O}_{F}$, which implies that $\left\langle\mathcal{L}_{i}\right\rangle=\operatorname{Ker} \hat{q}$ for the dual isogeny $\hat{q}: \hat{E} \rightarrow \hat{F}$ of $q$. Therefore, the subgroup $\left\langle\mathcal{L}_{i}\right\rangle$ of $\hat{E}$ does not depend on the choice of $i$. In particular, we have an inclusion

$$
\left\{S_{i} \mid i \in(\mathbb{Z} / m \mathbb{Z})^{*}\right\} / \cong \hookrightarrow\left\{\mathbb{P}\left(\mathcal{O}_{E} \oplus \mathcal{L}_{1}^{i}\right) \mid i \in(\mathbb{Z} / m \mathbb{Z})^{*}\right\} / \cong .
$$

We will see below that these sets actually coincide by checking their cardinality. Let us start the following claim.

Claim 3.1. Take a line bundle $\mathcal{L} \in{ }_{m} \hat{E}$. For $i, j \in(\mathbb{Z} / m \mathbb{Z})^{*}, \mathbb{P}\left(\mathcal{O}_{E} \oplus \mathcal{L}^{i}\right) \cong$ $\mathbb{P}\left(\mathcal{O}_{E} \oplus \mathcal{L}^{j}\right)$ if and only if there is a group automorphism $\psi_{1} \in \mathrm{Aut}_{0} E$ such that $\psi_{1}^{*} \mathcal{L} \cong \mathcal{L}^{ \pm i^{-1} j}$ holds. Consequently, the cardinality of the right hand side of (7) is $\varphi(m) /\left|H_{\hat{E}}^{\mathcal{L}_{1}}\right|$.

Proof. Since each of $\mathbb{P}\left(\mathcal{O}_{E} \oplus \mathcal{L}^{i}\right)$ and $\mathbb{P}\left(\mathcal{O}_{E} \oplus \mathcal{L}^{j}\right)$ has a unique $\mathbb{P}^{1}$-bundle structure, any isomorphism $\psi: \mathbb{P}\left(\mathcal{O}_{E} \oplus \mathcal{L}^{i}\right) \rightarrow \mathbb{P}\left(\mathcal{O}_{E} \oplus \mathcal{L}^{j}\right)$ induces an automorphism $\psi_{1}$ of $E$, which is compatible with $\psi$. We can see by Ha77, II. Ex. 7.9(b)] that $\psi_{1}$ satisfies the desired property. The opposite direction also follows from [ibid.].

We also have the following.

Claim 3.2. For $i, j \in(\mathbb{Z} / m \mathbb{Z})^{*}, S_{i} \cong S_{j}$ if and only if there is a group automorphism $\phi_{1} \in \mathrm{Aut}_{0} F$ such that $\phi_{1}(a)=\left( \pm i^{-1} j\right) \cdot$ a holds. Consequently, the cardinality of the left hand side of (77) is $\varphi(m) /\left|H_{F}^{a}\right|$.

Proof. Suppose that there is an isomorphism $\psi: S_{i} \rightarrow S_{j}$. As in the proof of Claim 3.1. $\psi$ induces an automorphism $\psi_{1}$ of $E$ which is compatible with $\psi$. It is also satisfied that the dual isogeny $\hat{\psi}_{1}$ preserves the subgroup $\left\langle\mathcal{L}_{1}\right\rangle$ of $\hat{E}$, and hence $\psi_{1}$ lifts an automorphism $\phi_{1}$ of $F \cong \hat{F} \cong E /\left\langle\mathcal{L}_{1}\right\rangle$. Since the left square in (6) is a fiber product, $\psi$ lifts to an automorphism $\phi$ of $F \times \mathbb{P}^{1}$. We can see that $\phi$ is of the form $\phi_{1} \times \phi_{2}$ for some $\phi_{2} \in A u t \mathbb{P}^{1}$. Since any translation on $F$ descends to a translation on $E$, replacing $\phi_{1}$ if necessary, we may assume that $\phi_{1} \in \operatorname{Aut}_{0} F$. Since $\phi$ descends to $\psi$, it should satisfy

$$
\phi \circ \rho_{i}(g)=\rho_{j}\left(g^{k}\right) \circ \phi
$$

for any $g \in G$ and some $k \in \mathbb{Z}$. By observing the action on $\mathbb{P}^{1}$, we know that $k=1$ or $m-1$, and moreover

$$
\phi_{2}(y)= \begin{cases}\lambda y & (\text { in the case } k=1) \\ \lambda / y & (\text { in the case } k=m-1)\end{cases}
$$


for $y \in \mathbb{P}^{1}$ and some $\lambda \in \mathbb{C}^{*}$. In the former case, we obtain that $\phi_{1}(a)=$ $\left(i^{-1} j\right) \cdot a$ holds, and in the latter case, $\phi_{1}(a)=\left(-i^{-1} j\right) \cdot a$ holds.

For $m \leq 3$, we can easily see from Claims 3.1 and 3.2 that the both side of (7) coincide. And hence, suppose that $m>3$. Then, it follows from Lemma 2.4. Claims 3.1 and 3.2 that the both side of (77) coincide:

$$
\begin{aligned}
& \left\{S_{i} \mid i \in(\mathbb{Z} / m \mathbb{Z})^{*}\right\} / \cong \\
= & \left\{\mathbb{P}\left(\mathcal{O}_{E} \oplus \mathcal{L}_{1}^{i}\right) \mid i \in(\mathbb{Z} / m \mathbb{Z})^{*}\right\} / \cong .
\end{aligned}
$$

The cardinality of this set is $\varphi(m) /\left|H_{\hat{E}}^{\mathcal{L}_{1}}\right|$.

Claim 3.3. In the above notation, $S_{i} \cong J_{S_{1}}(i)$ for all $i$ with $i \in(\mathbb{Z} / m \mathbb{Z})^{*}$.

Proof. Take an element $j \in(\mathbb{Z} / m \mathbb{Z})^{*}$ such that $i j=1$. Henceforth, we identify $F$ and $\hat{F}$ as group schemes by (2). For the normalized Poincare bundle $\mathcal{P}_{0}$ given in $\$ 2.2$, we define

$$
\mathcal{P}:=\mathcal{P}_{0} \otimes p_{1}^{*} \mathcal{O}_{F}(i O) \otimes p_{2}^{*} \mathcal{O}_{F}(j O) .
$$

Here, we regard elements $i, j \in(\mathbb{Z} / m \mathbb{Z})^{*}$ as integers satisfying $1 \leq i, j \leq$ $m-1$. Then the line bundle $\mathcal{P}$ satisfies

$$
\left.\mathcal{P}\right|_{x \times F} \cong \mathcal{O}_{F}(x+(j-1) O) \text { and }\left.\mathcal{P}\right|_{F \times y} \cong \mathcal{O}_{F}(y+(i-1) O)
$$

for any $x, y \in F$. Let us consider the commutative diagram:

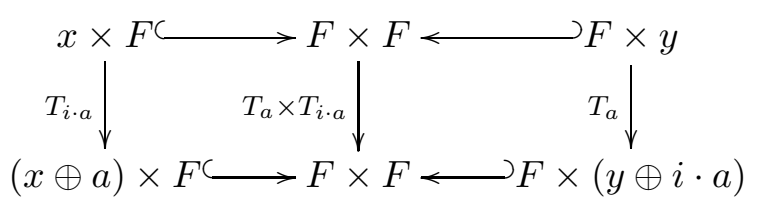

Here, the left vertical morphism is defined by the composition of morphisms

$$
x \times F \cong F \stackrel{T_{i \cdot a}}{\longrightarrow} F \cong(x \oplus a) \times F
$$

and similarly, the right vertical arrow is also defined by $T_{a}$. Now we have

$$
\begin{aligned}
\left.\left(\left(T_{a} \times T_{i \cdot a}\right)^{*} \mathcal{P}\right)\right|_{F \times y} & \cong T_{a}^{*}\left(\left.\mathcal{P}\right|_{F \times(y \oplus i \cdot a)}\right) \\
& \left.\cong \mathcal{P}\right|_{F \times(y \oplus i \cdot a)} \otimes \mathcal{O}_{F}(a-O)^{-i} \\
& \cong \mathcal{O}_{F}(y+i a-O) \otimes \mathcal{O}_{F}(a-O)^{-i} \\
& \cong \mathcal{O}_{F}(y+(i-1) O) \\
& \left.\cong \mathcal{P}\right|_{F \times y}
\end{aligned}
$$


Using ord $a=m$, we also have

$$
\begin{aligned}
\left.\left(\left(T_{a} \times T_{i \cdot a}\right)^{*} \mathcal{P}\right)\right|_{x \times F} & \cong T_{i \cdot a}^{*}\left(\left.\mathcal{P}\right|_{(x \oplus a) \times F}\right) \\
& \left.\cong \mathcal{P}\right|_{(x \oplus a) \times F} \otimes \mathcal{O}_{F}(i a-i O)^{-j} \\
& \cong \mathcal{O}_{F}(x+a+(j-2) O) \otimes \mathcal{O}_{F}(i a-i O)^{-j} \\
& \cong \mathcal{O}_{F}(x+(j-1) O) \\
& \left.\cong \mathcal{P}\right|_{x \times F}
\end{aligned}
$$

Hence, we obtain $\left(T_{a} \times T_{i \cdot a}\right)^{*} \mathcal{P} \cong \mathcal{P}$ by [Ha77, III. Ex. 12.4]. Let us define $\Delta_{\mathbb{P}^{1}}\left(\cong \mathbb{P}^{1}\right)$ to be the diagonal in $\mathbb{P}^{1} \times \mathbb{P}^{1}$. For the projection

$$
p_{1}:(F \times F) \times \Delta_{\mathbb{P}^{1}} \rightarrow F \times F,
$$

define a sheaf

$$
\mathcal{U}:=p_{1}^{*} \mathcal{P} .
$$

We regard $\mathcal{U}$ as a sheaf on $\left(F \times \mathbb{P}^{1}\right) \times\left(F \times \mathbb{P}^{1}\right)$. Then for any $g \in G$, we have

$$
\begin{aligned}
\left.\left(\left(\rho_{1}(g) \times \rho_{i}(g)\right)^{*} \mathcal{U}\right)\right|_{x \times \mathbb{P}^{1} \times y \times \mathbb{P}^{1}} \cong\left(\rho_{\mathbb{P}^{1}}(g) \times \rho_{\mathbb{P}^{1}}(g)\right)^{*}\left(\left.\mathcal{U}\right|_{\left.(x \oplus a) \times \mathbb{P}^{1} \times(y \oplus i \cdot a) \times \mathbb{P}^{1}\right)}\right. & \cong \rho_{\mathbb{P}^{1}}(g)^{*} \mathcal{O}_{\Delta_{\mathbb{P} 1}} \\
& \cong \mathcal{O}_{\Delta_{\mathbb{P}} 1} \\
& \left.\cong \mathcal{U}\right|_{x \times \mathbb{P}^{1} \times y \times \mathbb{P}^{1}}
\end{aligned}
$$

for $x, y \in F$, and

$$
\begin{aligned}
\left.\left(\left(\rho_{1}(g) \times \rho_{i}(g)\right)^{*} \mathcal{U}\right)\right|_{F \times z \times F \times z} & \cong\left(T_{a} \times T_{i \cdot a}\right)^{*}\left(\left.\mathcal{U}\right|_{F \times \zeta z \times F \times \zeta z}\right) \\
& \cong\left(T_{a} \times T_{i \cdot a}\right)^{*} \mathcal{P} \\
& \cong \mathcal{P} \\
& \left.\cong \mathcal{U}\right|_{F \times z \times F \times z}
\end{aligned}
$$

for any $z \in \mathbb{P}^{1}$, and note that both of $\left.\left(\left(\rho_{1}(g) \times \rho_{i}(g)\right)^{*} \mathcal{U}\right)\right|_{F \times z_{1} \times F \times z_{2}}$ and $\left.\mathcal{U}\right|_{F \times z_{1} \times F \times z_{2}}$ are zero for $z_{1} \neq z_{2} \in \mathbb{P}^{1}$, since $\operatorname{Supp} \mathcal{U}=(F \times F) \times \Delta_{\mathbb{P} 1}$. Then, by [Ha77, III. Ex. 12.4] we can check $\left(\rho_{1}(g) \times \rho_{i}(g)\right)^{*} \mathcal{U} \cong \mathcal{U}$, equivalently

$$
\left(\rho_{1}(g) \times \operatorname{id}_{F \times \mathbb{P}^{1}}\right)^{*} \mathcal{U} \cong\left(\operatorname{id}_{F \times \mathbb{P}^{1}} \times \rho_{i}\left(g^{-1}\right)\right)^{*} \mathcal{U} .
$$

This implies that

$$
\left(q_{1} \times \operatorname{id}_{F \times \mathbb{P}^{1}}\right)_{*} \mathcal{U} \cong\left(\operatorname{id}_{S_{1}} \times \rho_{i}\left(g^{-1}\right)\right)^{*}\left(q_{1} \times \operatorname{id}_{F \times \mathbb{P}^{1}}\right)_{*} \mathcal{U},
$$

that is, the sheaf $\left(q_{1} \times \operatorname{id}_{F \times \mathbb{P}^{1}}\right)_{*} \mathcal{U}$ is $G$-invariant with respect to the diagonal action of $G$ on $S_{1} \times\left(F \times \mathbb{P}^{1}\right)$, induced by the trivial action on $S_{1}$ and $\rho_{i}$ 
on $F \times \mathbb{P}^{1}$. Since $G$ is cyclic, we can conclude that $\left(q_{1} \times \operatorname{id}_{F \times \mathbb{P}^{1}}\right)_{*} \mathcal{U}$ is $G$-equivariant, and hence there is a coherent sheaf $\mathcal{U}^{\prime}$ on $S_{1} \times S_{i}$ such that

$$
\left(q_{1} \times \operatorname{id}_{F \times \mathbb{P}^{1}}\right)_{*} \mathcal{U} \cong\left(\operatorname{id}_{S_{1}} \times q_{i}\right)^{*} \mathcal{U}^{\prime} .
$$

For $x \times z \in F \times \mathbb{P}^{1}$, we have $\left.\left.\mathcal{U}\right|_{F \times z \times x \times z} \cong \mathcal{P}\right|_{F \times x}$, which is a line bundle of degree $i$ on $F$ by (9). The isomorphism (10) yields

$\left.\left.\mathcal{U}^{\prime}\right|_{S_{1} \times q_{i}(x \times z)} \cong\left(\left(q_{1} \times \operatorname{id}_{F \times \mathbb{P}^{1}}\right)_{*} \mathcal{U}\right)\right|_{S_{1} \times(x \times z)} \cong\left(q_{1} \times \operatorname{id}_{F \times \mathbb{P}^{1}}\right)_{*}\left(\left.\mathcal{U}\right|_{\left(F \times \mathbb{P}^{1}\right) \times(x \times z)}\right)$.

Here, the second isomorphism follows from [BO95, Lemma 1.3] and the smoothness of $q_{1}$. Since $\left.\mathcal{U}\right|_{\left(F \times \mathbb{P}^{1}\right) \times(x \times z)}$ is actually a sheaf on $F \times z \times x \times z$ and the restriction $\left.q_{1}\right|_{F \times z}$ is isomorphic for $z \in \mathbb{P}^{1} \backslash\{0, \infty\},\left.\mathcal{U}^{\prime}\right|_{S_{1} \times q_{i}(x \times z)}$ is also a line bundle of degree $i$ on $F_{z} \times q_{i}(x \times z)$ for such $z$. Here, $F_{z}(\cong F)$ is a fiber of $\pi_{1}$ over the point $q_{\mathbb{P}^{1}}(z)$. Then, by the universal property of $J_{S_{1}}(i)$, there is a morphism between the open subsets of $S_{i}$ and $J_{S_{1}}(i)$ over $\mathbb{P}^{1} \backslash\left\{q_{\mathbb{P}^{1}}(0), q_{\mathbb{P}^{1}}(\infty)\right\}$. Since $\left.\left.\mathcal{U}^{\prime}\right|_{S_{1} \times q_{i}(x \times z)} \not \mathcal{U}^{\prime}\right|_{S_{1} \times q_{i}(y \times z)}$ on $F_{z}$ for $x \neq y \in$ $S_{i}$, this morphism is injective, and hence $S_{i}$ and $J_{S_{1}}(i)$ are birational over $\mathbb{P}^{1}$. Then, [BHPV, Proposition III. 8.4] implies the result.

Now, we obtain the following.

Proposition 3.4. Let $E$ be an elliptic surface, and define $S$ to be an elliptic ruled surface $\mathbb{P}\left(\mathcal{O}_{E} \oplus \mathcal{L}\right)$ for a line bundle $\mathcal{L} \in{ }_{m} \hat{E}$ for $m>0$. Then we have

$$
\operatorname{FM}(S)=\left\{\mathbb{P}\left(\mathcal{O}_{E} \oplus \mathcal{L}^{i}\right) \mid i \in(\mathbb{Z} / m \mathbb{Z})^{*}\right\} / \cong .
$$

This set consists of $\varphi(m) /\left|H_{\hat{E}}^{\mathcal{L}}\right|$ elements. In the case $m>3$, the cardinality $\left|H_{\hat{E}}^{\mathcal{L}}\right|$ is 2,4 or 6 , depending on the choice of $\hat{E}$ and $\mathcal{L}$.

Proof. The first statement is a direct consequence of Theorem 2.1, the equation (8) and Claim 3.3. The second is a direct consequence of Claim 3.1. We can compute the cardinality of $H_{\hat{E}}^{\mathcal{L}}$ by Lemmas 2.3 and 2.4 ,

We are in a position to show Theorem 1.1 .

Proof of Theorem 1.1. The condition $|\operatorname{FM}(S)| \neq 1$ implies that $S$ has an elliptic fibration $\pi: S \rightarrow \mathbb{P}^{1}$ (see [BM01). Hence, either of the cases (1-i), (1-ii) or (ii) in Theorem 2.5 occurs (recall that we work over $\mathbb{C}$ ). In each case, we see from Remark 2.6 that $\lambda_{S}=1, m$ and 2 respectively. It follows from Claim 2.2 that $S$ actually fits into the case (1-ii) with $m>4$. Now set $S=\mathbb{P}\left(\mathcal{O}_{E} \oplus \mathcal{L}\right)$ for some $\mathcal{L} \in{ }_{m} \hat{E}$ for some $m>4$. Then the assertion follows from Proposition 3.4 . 


\section{Further questions}

\subsection{Autoequivalences}

Let $S:=\mathbb{P}\left(\mathcal{O}_{E} \oplus \mathcal{L}\right)$ be an elliptic ruled surface with non-trivial FourierMukai partners, where $E$ is an elliptic curve, and $\mathcal{L} \in{ }_{m} E$ for some $m>4$, as in Theorem 1.1. Then, the group $H_{S}$ defined in $\$ 2.1$ coincides with the group $H_{\hat{E}}^{\mathcal{L}}$, by the results in $₫ 3$, Note that there are no $(-2)$-curves on $S$, and hence no twist functors associated with $(-2)$-curves appears in Auteq $D(S)$. Moreover, we can see that the $\mathbb{P}^{1}$-bundle $f: S \rightarrow E$ has two sections $C_{0}$ and $C_{1}$, and $m C_{0}$ and $m C_{1}$ are the multiple fibers of $\pi$. We can also check that

$$
\left\langle\mathcal{O}_{S}(D) \mid D \cdot F=0\right\rangle=\left\langle\mathcal{O}_{S}\left(C_{0}\right), \mathcal{O}_{S}\left(C_{1}\right)\right\rangle
$$

in $\operatorname{Pic}(S)$, where $F$ is a smooth fiber of $\pi$. Therefore, by the main theorem of [Ue15], we have the following short exact sequence:

$$
\begin{aligned}
1 \rightarrow\left\langle\otimes \mathcal{O}_{S}\left(C_{0}\right), \otimes \mathcal{O}_{S}\left(C_{1}\right)\right\rangle & \rtimes \text { Aut } S \times \mathbb{Z}[2] \rightarrow \text { Auteq } D(S) \\
& \rightarrow\left\{\left(\begin{array}{ll}
c & a \\
d & b
\end{array}\right) \in \Gamma_{0}(m) \mid b \in H_{\hat{E}}^{\mathcal{L}}\right\} \rightarrow 1 .
\end{aligned}
$$

Here for an integer $b$, coprime with $m$, we again denote by $b$ the corresponding element in $H_{\hat{E}}^{\mathcal{L}}\left(\subset(\mathbb{Z} / m \mathbb{Z})^{*}\right)$, and $\Gamma_{0}(m)$ is the congruence subgroup of $\mathrm{SL}(2, \mathbb{Z})$, defined in Ue15.

Since other ruled surfaces with an elliptic fibration has no non-trivial Fourier-Mukai partners, the description of their autoequivalence groups is directly given by [Ue15].

For elliptic ruled surfaces without elliptic fibrations, a description of the autoequivalence group will be given in the forthcoming paper [Ue].

\subsection{Positive characteristic}

The proof of Theorem 1.1 does not work over positive characteristic fields. We finish this section to raise the following:

Problem 4.1. (i) In the notation of 92.3 , consider the case $e=-1$ and $p=2$. Study when $S$ has an elliptic fibration, and if it has, study the singular fibers of the fibration.

(ii) Describe the set $\operatorname{FM}(S)$ for elliptic ruled surfaces $S$ over a positive characteristic field.

In [Ma71, Theorem 4], Maruyama states that in the case $e=-1$ and $p=2, S$ ( $\mathbf{P}_{1}$ in his notation) has an elliptic fibration. But it seems to the author that he gave no proof of this statement. See also [Ma71, Remark 7].

Furthermore, in the case (i-5) in Theorem [2.5, if $p \geq 5, S$ may have non-trivial Fourier-Mukai partners, since $\lambda_{S}=p$ (we omit the proof of this 
fact here). It is also an interesting question to describe $\operatorname{FM}(S)$ in this case. [KU85, Examples 4.7, 4.8] fit into the case (i-5). To show Theorem 1.1, the equality (8) was a key. The author believes that the description in KU85, Examples 4.7, 4.8] should be useful to describe $\operatorname{FM}(S)$ for $S$ in the case (i-5).

\section{References}

[BO95] A.I. Bondal, D.O. Orlov, Semiorthogonal decomposition for algebraic varieties, alg-geom 9712029 .

[BHPV] Barth, Wolf P.; Hulek, Klaus; Peters, Chris A. M.; Van de Ven, Antonius, Compact complex surfaces. Second edition. Results in Mathematics and Related Areas. 3rd Series. A Series of Modern Surveys in Mathematics, 4. Springer-Verlag, Berlin, 2004. xii+436 pp.

[Br98] T. Bridgeland, Fourier-Mukai transforms for elliptic surfaces. J. Reine Angew. Math. 498 (1998), 115-133.

[BM01] T. Bridgeland, A. Maciocia, Complex surfaces with equivalent derived categories. Math. Z. 236 (2001), 677-697.

[Ha77] R. Hartshorne, Algebraic Geometry, Springer-Verlag, Berlin Heidelberg New York, 1977.

[KU85] T. Katsura, K. Ueno, On elliptic surfaces in characteristic $p$, Math. Ann. 272, 291-330 (1985).

[Ka02] Y. Kawamata, D-equivalence and K-equivalence. J. Differential Geom. 61 (2002), 147-171.

[Ma71] M. Maruyama, On automorphism groups of ruled surfaces, J. Math. Kyoto Univ. 11 (1971), 89-112.

[Or97] D. Orlov, Equivalences of derived categories and K3 surfaces. Algebraic geometry, 7. J. Math. Sci. (New York) 84 (1997), 1361-1381.

[To11] T. Togashi, "Daen fibration wo motsu seihyousuu no sensiki kyokumen ni tsuite" (in Japanese), Master's Thesis, Tokyo Metropolitan University, (2011).

[Ue04] H. Uehara, An example of Fourier-Mukai partners of minimal elliptic surfaces. Math. Res. Lett. 11 (2004), no. 2-3, 371-375.

[Ue11] H. Uehara, A counterexample of the birational Torelli problem via Fourier-Mukai transforms. J. Algebraic Geom. 21 (2012), no. 1, 7796. 
[Ue15] H. Uehara, Autoequivalences of derived categories of elliptic surfaces with non-zero Kodaira dimension, arXiv:1501.06657.

[Ue] H. Uehara, Autoequivalences of derived categories of surfaces with non-torsion canonical divisor. (in preparation)

Department of Mathematics and Information Sciences, Tokyo Metropolitan University, 1-1 Minamiohsawa, Hachioji-shi, Tokyo, 192-0397, Japan e-mail address : hokuto@tmu.ac.jp 